\title{
Effect of Fluoride Ions on the Microanatomy of Lungs in Albino Rats.
}

\author{
Bashir Ahmad Shah MS**, Gh.Mohd.Bhat MS***, Mohd.Saleem Itoo MD**, \\ Nasseer Ahmad Shah MD*, Shaheen Shadad MD****, \\ Javed Ahmad Khan MD*, \\ Department of Anatomy, Government Medical College, Srinagar. \\ Lecturer* Assistant professor**, Associate professor***, Professor****
}

\begin{abstract}
The present study was conducted to study the effect of graded doses of Sodium fluoride on the microanatomy of lungs of Albino rats over different periods of time. Sixty male adult albino rats taken from the animal house of Govt. medical College Srinagar were divided randomly into four groups of 15 animals each. Animals of the first three groups were given fluorinated water in various concentrations to drink and fourth group served as the control group getting plain tap water to drink. Animals from each group were sacrificed and examined after 30, 60, and 90 days of therapy and gross and microscopic changes recorded. It was observed that fluorides induce dose and duration dependent microscopic changes in lung tissue ranging from mild edema to gross necrosis.
\end{abstract}

Key Words: Sodium Fluoride, Edema, Necrosis, Fluorosis, Fatty infiltration

\section{Introduction:}

Fluoride is any combination of elements containing the fluoride ion. In its elemental form, fluorine is a pale yellow, highly toxic and corrosive gas. In nature, fluorine is found combined with minerals as fluorides. It is the most chemically active nonmetallic element of any element and also is the most reactive electro-negative ion. Because of this extreme reactivity, fluorine isn't found in nature as an uncombined element. Fluorine is a member of group VIIa of the periodic table. It readily displaces other halogens - such as chlorine, bromine and iodine - from their mineral salts. With hydrogen it forms hydrogen fluoride gas which, in a water solution, becomes hydrofluoric acid. It is total fluoride intake from all sources which must be considered for any adverse health effect evaluation, including intake by ingestion, inhalation and absorption through the skin. In 1971, the World Health Organization (WHO) stated: "In the assessment of the safety of a water supply with respect to the fluoride concentration, the total daily fluoride intake by the individual must be considered."

Household exposures to fluorides can occur with the use of Teflon pans, fluorine-based products, insecticides sprays and even residual airborne fluorides from fluoridated drinking water. Besides fluoridated water and toothpaste, many foods contain high levels of fluoride compounds due to pesticide applications. Grape juice was found to contain more than $6.8 \mathrm{ppm}$ fluoride Soy infant formula is high in both fluoride and aluminum, far surpassing the "optimal" dose - even when de-fluoridated water is used in preparation — and has been shown to be a significant risk factor in dental fluorosis.

The total intake from these sources alone already greatly exceeds the levels known to cause the third stage of skeletal fluorosis. Prolonged total Fluoride intake exceeding $1.0 \mathrm{mg} / \mathrm{day}$ can produce clinical signs of fluorosis in adults. Generally speaking, human fluoride toxicity will manifest as any combination of the following: Dental Fluorosis: the most obvious and easily diagnosed form of Dental Fluorosis is usually caused by over-exposure to Fluoride when the dental enamel is actively mineralizing during early childhood.

Skeletal Fluorosis: involves abnormal mineralization of bone and soft tissues and/or the disruption of normal activity of the osteocytes. For this reason, Skeletal Fluorosis often mimics "generic" osteoarthritis and/or osteoporosis in relatively young adults.

Systemic Fluorosis: due to the chemical nature of fluoride and its action(s) within mammalian systems which are not limited to teeth and bone, Fluoride toxicity may potentially be linked to every major multiple cause ailment of the 20th century from cancer to Attention Deficit Disorder.

Fluoride toxicity may be acute or chronic, with effects ranging from cosmetic damage, to disability and even death. With the exception of Dental Fluorosis, Fluoride-related illness is often attributed to other diseases or syndromes (i.e. osteoarthritis for Skeletal Fluorosis, cardiovascular failure for death by acute Fluoride poisoning) making Fluorosis in itself very difficult to track epidemiologically in the absence of an ecosystem health framework." 
Though apparently vague and non-specific, most of the symptoms of Fluoride toxicity point towards some kind of profound metabolic dysfunction, and are strikingly similar to the symptoms of Hypothyroidism. Even in small quantities, sodium fluoride is a deadly poison to which no effective antidote has been found.

Many prescription drugs contain a hidden dose of fluoride. Several fluoride-containing medications have been banned because they caused death and illness. Fluoride is highly toxic to the liver. In the liver, fluoride interferes with the metabolism of thyroid hormones; it creates thyroid disorders and associated diseases that affect muscles, heart, immune system, etc. Many psychiatric drugs contain fluoride. Silicon fluoride is linked to antisocial behavior in youth. The fluorine ion is an enzyme inhibitor. The one side effect common to fluorinated drugs is memory loss. These drugs, including Prozac (fluoxetene)--and Paxil--antidepressant drugs, contain three fluorine atoms in each molecule that kill enzymes in the brain that normally maintain mood stability. More than 50 percent of infants are currently formula fed by 1 month of age, and these infants are likely to be continuously exposed to high intakes of fluoride for 9 or 10 months - a circumstance quite rare in the 1960s and early 1970s."Fomon et al (1970). ${ }^{3}$

In any case, fluoride is unavoidable in the diet, since it is the thirteenth most abundant element and is extremely reactive, forming many compounds available to the human body. Fluorine occurs in nature as calcium fluoride. Sodium fluoride is an industrial waste product from the aluminum and phosphate fertilizer industries. Since the 1920s and 1930s, it has been sold as a potent roach and rat killer. Sodium fluoride (NaF), is over five hundred times more soluble than calcium fluoride $\left(\mathrm{CaF}_{2}\right)$. It requires eighty times more $\mathrm{CaF}_{2}$ to kill a rat than $\mathrm{NaF}$. Fluoridation critics never accepted the transformation of sodium fluoride from dangerous chemical to benign cavity fighter. Fluoride depletes calcium from the body.

Twentieth century was considered to be the age of industrialization but unfortunately rapid industrial growth has resulted in complex range of health problems due to environmental pollution and one of the most important health hazards of environmental pollution is "Fluorosis".

The main clinical signs of Fluorosis are manifest in the skeletal system but there are direct and indirect toxic effects on other systems of the body including Nervous, Urinary, Reproductive, Circulatory System and Respiratory System including lungs.

\section{Material And Methods}

A total of 60 male adult albino rats were randomly selected and divided into four groups of 15 animals each. Group A: The animals of this group were given drinking water with $10 \mathrm{ppm}$ concentration of fluoride besides standard diet. Group B: The animals of this group were given drinking water with $500 \mathrm{ppm}$ concentration of fluorides besides standard diet. Group C: The animals of this group were given drinking water with $1000 \mathrm{ppm}$ concentration of fluoride besides standard diet. Group D: The animals of this group were given plain tap water to drink besides standard diet. This served as the control group. Fluorinated water was prepared by dissolving sodium in tap water. Addition of one mg of sodium fluoride to one liter of water makes a concentration of one part per million (ppm). The animals were observed daily for changes in appearance and body weight. Animals of different groups were studied after 30, 60, and 90 days of therapy when 15 animals from each of the four groups were sacrificed and examined. At the time of each examination the animals were weighed and anaesthetized by chloroform, a midline incision was given and lungs were dissected out and put on a dish containing Formalin. Macroscopic changes if any were observed and compared with the control group. The lungs were subjected to processing in an automatic tissue processor, sections 5 micrometer thick were cut with a rotator microtome, stained with Haematoxylin \&Eosin stain and observed under compound light microscope.

\section{Observations}

On gross examination the lungs of both control and experimental group of animals were normal in appearance. However in the animals of group B and C after 60 and 90 days of therapy the color of the lungs was slightly lighter and discoloration patches were visible.

Microscopic changes: The lungs of group A showed minimal microscopic changes after 30 days of treatment. The cellular architecture of the lungs of this group was maintained even after 60 and 90 days of therapy but mild edema and occasional hemorrhages were seen (Fig No.1). The lungs of group B after 30 days of treatment showed edema and frequent hemorrhages. However after 60 and 90 days of therapy occasional necrosis and fibrosis was also seen (Fig.No.2 \& 4). The animals of group C were the worst affected. After 30 days of treatment, the lungs of this group showed edema, frequent hemorrhages and occasional necrosis. After 60 days of therapy the lungs of this group showed distortion of the lung parenchyma with frequent hemorrhages and necrosis. The lungs of the animals of this group after 90 days of treatment were the worst affected and hardly any normal lung parenchyma was visible. The histological architecture of the lung tissue was distorted and frequent areas of hemorrhages, necrosis and fatty infiltration were found (Fig.No.3). Fibrosis was also seen in some cases. 


\section{Discussion}

The present study was aimed at evaluating the effects of varying strengths of Sodium Fluoride on the lungs of Albino Rats. A notable reduction in the body weight of experimental animals was seen after Fluoride administration which was more obvious in the animals of higher dose group. It is obvious that fluoride toxicity causes metabolic and structural changes which in turn cause wasting of muscle mass and loss of body weight. With low concentrations of fluorides the histological architecture of lung parenchyma was maintained but there were occasional haemorrhages and edema of the lung tissue. With increased concentration and duration of exposure to fluorides frequent hemorrhages, edema of lung tissue, distortion of cell outline, small areas of necrosis and hemorrhages, diffuse areas of necrosis, fatty infiltration (Fig.No.3) and finally fibrosis was observed. Alfred Taylor et al (1961) ${ }^{1}$ and Simon et al $(1968)^{11}$ have also reported weight loss in the animals fed on fluorides. Weight loss in fluorotic humans was reported by Short et al $(1937)^{9}$ and Sidiqui $(1955)^{10}$.

The involvement of lungs in fluorosis has not received due attention during the previous years .However during the past few years attention has been focused on the various adverse effects of fluorides on this organ of vital importance. Most of the previous studies have focused their attention on the physiological effects of fluorides on the lungs.

It was Himwich $\mathrm{H}$ E et al $(1942)^{5}$ who stated that fluorine may cause anoxia in the newborn and shorten the period of their survival. The $24^{\text {th }}$ edition of U S Dispensary (1950) reports fluorides cause respiratory failure. G J Cox et al $(1950)^{4}$ observed that fluorides cause acute poisoning and the changes are comparable to those seen in high grade anoxia. Zajusz $(1966)^{12}$ noticed that calcium fluoride injected intratrachealy provoked inflammation and degeneration of the respiratory epithelium associated with enlargement of lymph nodes and hemorrhages. Martin Drodz et al (1981) ${ }^{7}$ observed that fluorides decrease the skin and lung collagen levels. M Humickzwaska et al (1944) ${ }^{6}$ reported that lungs of the animals exposed to fluorides showed numerous acidophilic leucocytes in the interstitial tissue and an overall increase in the number of lymphoid cells. An increase in the number of pneumocytes and macrophages was also observed. Ayedin $\mathrm{G}$ et al $(2003)^{2}$ studied the possible effects of multigenerational administration of sodium fluoride $(\mathrm{NaF})$ via drinking water on lung tissue morphology and biochemistry. He studied second-generation adult male rats and histological findings showed alveolar congestion, alveolar cell hyperplasia and necrosis, prominent alveolar septal vessels, epithelial desquamation and macrophages in the alveolar spaces in the experimental groups. Additionally, there were inflammatory infiltrations in peribronchial, perivascular, intraparenchymal and respiratory tract lumen; intraparenchymal hyperaemic vessels; respiratory epithelial desquamation and proliferation; intraparenchymal thick walled vessels; parenchymal fibrosis; bronchiolitis; pneumonic and focal emphysematous areas were also seen. Furthermore, the lung parenchyma was observed to have a distorted appearance with loss of alveolar architecture. Oncu M et al (2006) ${ }^{8}$ studied to investigate the lipid peroxidation and histological effects of chronic fluorosis on first and second generation rat lung tissues. It was found that the plasma fluoride and the lung TBARS levels of fluoride supplemented F1 and F2 rats were higher than controls. There were marked histological changes in the lung tissues of fluoride supplemented F1 and F2 rats, as follows: in F1 rats; loss of alveolar architecture, emphysematous areas, desquamation of alveolar epithelium and alveolar congestion were observed. There were thickened interalveolar septae and congestion of alveolar septal vessels. Intraparenchymal thick-walled vessels were also observed. There were markedly perivascular and intraparenchymal focal mononuclear cell infiltrations. In F2 rats, in addition to these changes, there were lipid cell hyperplasia and increased connective tissue mass in the parenchymal areas. It was concluded that chronic fluorosis causes a marked destruction in lung tissues of F1 and F2 rats by causing lipid peroxidation.

\section{Conclusion}

In the present study it was observed that fluorides have an obvious deleterious effect on the lung tissue. Various changes observed varied from edema, small hemorrhages in the lung tissue to lymphocytic infiltration, fatty infiltration, thickening of alveolar septa, thickening of vascular wall, complete destruction of lung architecture, fatty change and fibrosis. The changes depended upon the amount of fluorides in the drinking water and the duration of time for which the animals consumed fluorinated water. It is quiet obvious that fluorides cause structural damage to the lungs to such a large extent that functional impairment is unavoidable.

\section{References:}

[1]. Alfred Taylor, Nell Taylor. "Research Paper, 1965, "University of Texas".

[2]. Aydin G, Ciçek E, Akdoğan M, Gökalp O. J Appl Toxicol. 2003 Nov-Dec; 23(6):437-46.

[3]. Fomon SJ, Ekstrand J. Fluoride intake by infants. Journal of Public Health Dentistry, 1995, 59(4):229-34.

[4]. G J Cox, HC Hodge. Journal of American Dental Association, 1950, Vol 40: 440.

[5]. Himwich HE. American Journal Of Physiology 1942, Vol. 3, pp.203-205.

[6]. Humickzwaska M, Hermach U. Folia Biol Krakow 1994, 42 pp 157-166.

[7]. Martin Drodz. Toxicol. Euorop. Resi, 1981, Vol. 3 No. 5 pp.237-239-241.

[8]. Oncu M, Gulle K, Karaoz E, Gultekin F, Karaoz S, Karakoyun I, Mumcu EToxicol Ind Health. 2006. sOct;22(9):375-80

[9]. Shortt HE, Pandit CG,Raghavachari JL. "endemic Fluorosis” Ind. Med. Gaz.1937, 72-396, 137 
[10]. Sidiqui AH. "Endemic Fluorosis in Nalganda district of Hyderabad" Brit. Med. J. 1955, 1408-13.

[11]. Simon G Journal of Nutrition, 1968, 68: 152-156.

[12]. Zajusz K. "Effect of Fluoride on the Respiratory Tract of Rat" Proc. Pathol. Dows. Inst. Med. Pracy, 1966, 17/4, $287-295$.

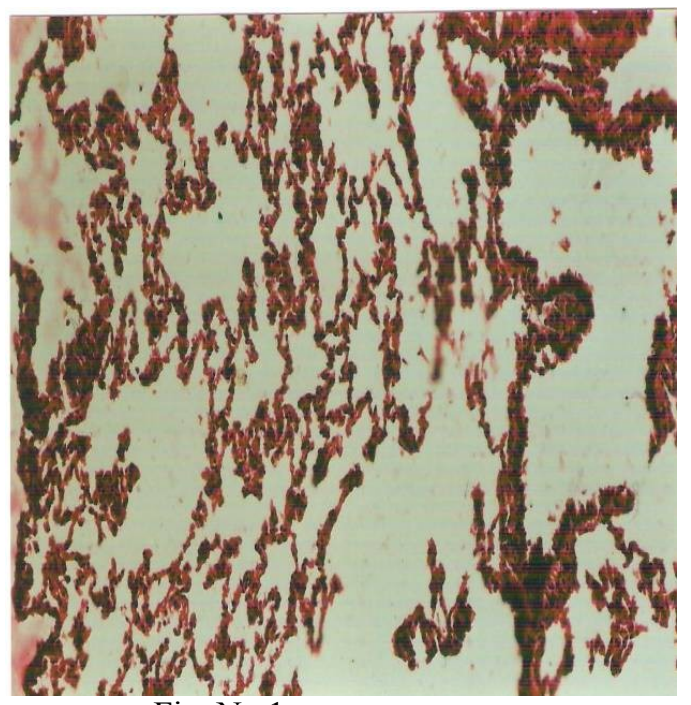

Fig. No.1

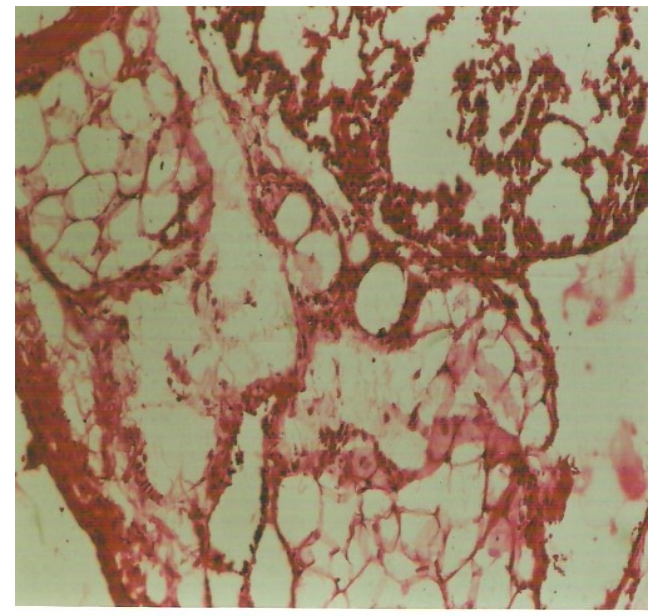

Fig. No. 3:

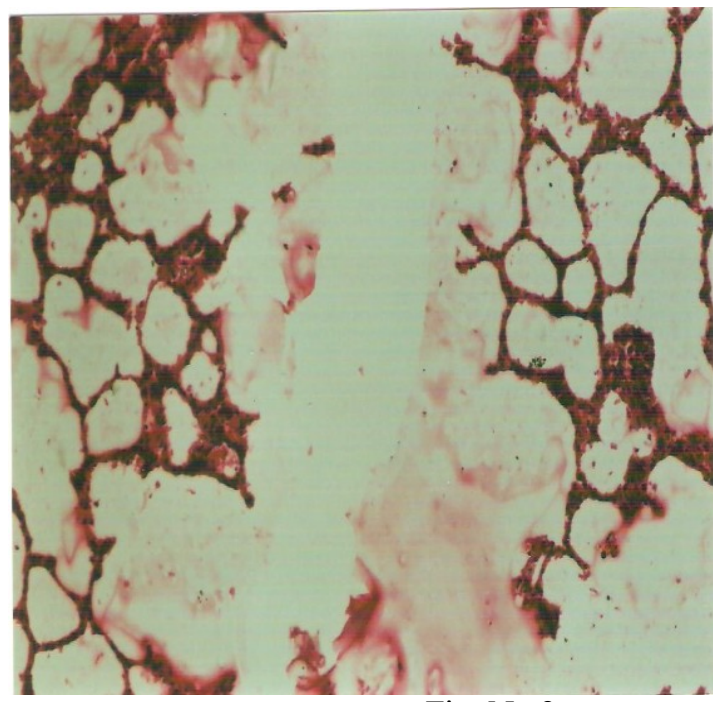

Fig. No.2

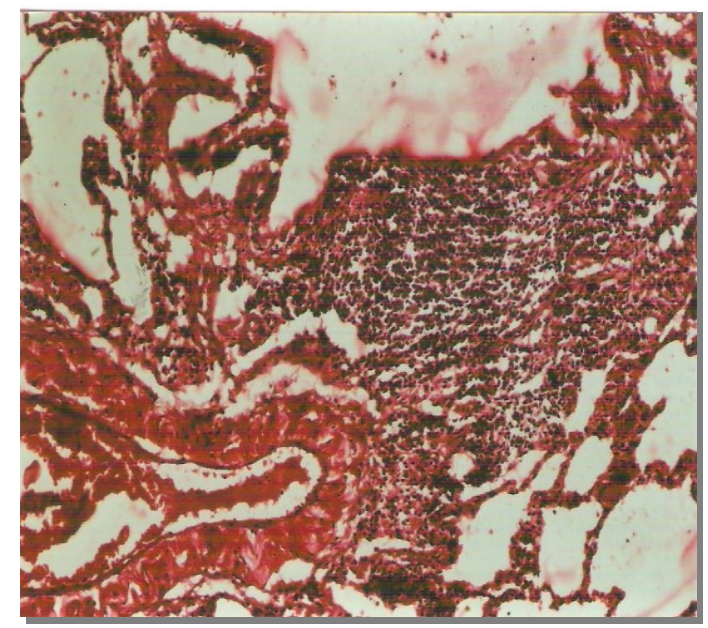

Fig. No. 4:

\section{Figure Legends:}

Fig. No. 1: Microphotograph of lungs of Group B after 60 days of treatment showing intra alveolar haemorrhages and alveolar destruction. Mag: 100X

Fig. No. 2: Microphotograph of lungs of Group A after 90 days of treatment showing thickening, lymphocytic infiltration and destruction of alveoli. Mag $100 X$

Fig. No. 3:Microphotograph of lungs of Group B after 90daysof therapy showing dense peribronchiolar infiltrate, and fatty infiltration, necrosis and fibrosis, with Mag: $100 \mathrm{X}$

Fig. No.4: Microphotogarph of lung of Group C after 90 days of therapy showing lymphocytic infiltraton, vessel wall thickening,necrosis of lung tissue and fibrosis. Mag.100X 